\title{
Determinación de arsénico en sedimento marino por espectrocopía atómica mediante su hidruro volátil generado electroquímicamente
}

Hugo Romero1, Lenys Fernández² Andrea Caiminagua', José Alvarado²

Universidad Técnica de Machala' Universidad Simón Bolívar, Venezuela² hromero@utmachala.edu.ec1

\section{Resumen}

El presente estudio describe la determinación de arsénico en sedimento marino de la Comuna de Bajo Alto, Provincia de El Oro, Ecuador. La generación electroquímica de este hidruro metálico volátil (arsina) utilizó como cátodo una amalgama Au/Hg (oro/mercurio), en una solución 0,5 $\mathrm{M}$ de $\mathrm{H} 2 \mathrm{SO} 4$. Los resultados se compararon con los obtenidos con otros cátodos utilizados comúnmente para la generación de este hidruro metálico. Se muestra que los sedimentos marinos de la comuna de Bajo Alto no están contaminados por arsénico (As) ya que la concentración de este metal fue de $0.05 \mathrm{mg} / \mathrm{Kg}$, mientras que los límites permitidos de acuerdo a la Organización Mundial de la Salud son de $5 \mathrm{mg} / \mathrm{Kg}$. Adicionalmente, el cátodo de Au/Hg tiene mejor tolerancia a la interferencia y mejor repetitividad que los cátodos de Au, Pt, Pb o carbono vítreo reticulado (RGC). La exactitud del método fue verificada determinando As en un material de referencia certificado.

Palabras clave: Generación electroquímica, hidruro volátil, arsénico, espectroscopía atómica.

\section{Abstract}

In this study, the determination of arsenic in marine sediment of Bajo Alto Commune, Province of El Oro, Ecuador is described. The electrochemical generation of this volatile metallic hydride (arsine), was carried out using an amalgam cathode Au / Hg (gold / mercury) in a 0.5M H2SO4 solution. The results were compared with those obtained with other commonly used cathodes for generating this metal hydride. The results show that the marine sediments are not contaminated by arsenic (As). The concentration of mercury was $0.05 \mathrm{mg} / \mathrm{kg}$, while the permitted limit according to the World Health Organization is $5 \mathrm{mg} / \mathrm{Kg}$. In addition, the cathode of $\mathrm{Au} / \mathrm{Hg}$ has better tolerance to interference and repeatability of the cathodes of $\mathrm{Au}, \mathrm{Pt}, \mathrm{Pb}$ or reticulated glass carbon (RGC). The accuracy of the method was verified by determining As in a certified reference material.

Keywords: Electrochemical generation, volatile hydride, arsenic, atomic spectroscopy.

Cómo citar este artículo: Romero H., Fernández L., Caiminagua A. y Alvarado J. (2015) Determinación de arsénico en sedimento marino por espectrocopía atómica mediante su hidruro volátil generado electroquímicamente. CUMBRES, Revista Científica. 1(2) $12-15$

\section{Cumbres}




\section{Introducción}

Los metales pesados constituyen uno de los contaminantes más importantes en el medio ambiente debido a su elevada persistencia y toxicidad. El problema de contaminación por metales, incluso a nivel de trazas, se ha incrementado en las últimas décadas debido al desarrollo industrial y a diversas actividades antropogénicas, produciendo efectos graves en la salud y medioambiente; los metales pesados de mayor toxicidad son Sb, As, $\mathrm{Ni}, \mathrm{Bi}, \mathrm{Tl}, \mathrm{Hg}$, Cd y Pb.

Los ríos constituyen una de las principales vías de transporte de metales a las zonas costeras, debido a la gran afinidad que tienen estos elementos para ser transportados en el material suspendido, siendo la costa el espacio más sensible a ser afectado, ya que los metales al entrar en contacto con la zona marina, sufren procesos que junto con otros factores ambientales, permiten su acumulación en los sedimentos.

Bajo Alto puede ser un foco de contaminación en sus sedimentos debido a que desembocan ríos tales como el Río Pagua, a quien se le atribuye contaminación por las zonas mineras aledañas al río. De igual manera se encuentra la desembocadura del Río Jubones, vinculando su contaminación a la zona agrícola.

La biodisponibilidad de metales en los sedimentos tiene una acción directa sobre algunas especies acuáticas, muchas de las cuales acumulan altas concentraciones con efectos crónicos. Estas especies pueden ser extraídas de algunos sectores de Bajo Alto y ser comercializadas sin saber los riesgos que conllevan para quienes las consumen, ocasionando efectos que puedan alterar la salud a largo plazo si la exposición ha sido a concentraciones muy bajas en períodos prolongados, e incluso la muerte si dichos organismos se encuentran expuestos a altas concentraciones.

La determinación de metales en los sedimentos es un buen indicador del origen de los contaminantes en el medio y de los impactos que éstos pueden producir en la biodiversidad marina.Los niveles excesivos de Arsénico en el medio marino afecta de manera directa a la vida marina, originando riesgos en la salud humana de quienes los utilizan como fuente de alimentación, pudiendo ocasionar efectos tóxicos a corto o largo plazo.

Los problemas de salud que genera la ingesta de este metal están asociados a daños sobre el intestino, corazón, sistema nervioso, hígado y piel. En una intoxicación aguda los principales síntomas son dolor abdominal, vómitos y diarrea; mientras que durante una intoxicación a largo plazo se manifiestan daños en la piel, como las lesiones cutáneas. Así mismo, una exposición prolongada puede causar cáncer de piel, inclusive ocasionar cáncer de vejiga y pulmón.

La Generación de Hidruros (HG) es un método de introducción de muestras, para la determinación de elementos formadores de hidruros volátiles, tales como: As, $\mathrm{Bi}, \mathrm{Ge}, \mathrm{Pb}$, $\mathrm{Cd}, \mathrm{Tl}, \mathrm{Sb}, \mathrm{Se}, \mathrm{Sn}, \mathrm{Te}$, que consiste en la transformación del analito previamente oxidado el cual es posteriormente reducido en la formación de su respectivo hidruro, por medio de una reacción química o electroquímica.

Existen dos vías para la formación de hidruros. La primera implica la reacción química entre el analito en estado oxidado y un agente reductor, conocida como Generación Química de Hidruros (CHG). La otra vía, conocida como Generación Electroquímica de Hidruros (EcHG), implica la reducción del analito mediante un flujo de electrones seguida de la reacción con átomos de hidrógeno, presentes en el medio de reacción, en la superficie del cátodo de una celda electroquímica.

En la presente investigación se analizaron los niveles de Arsénico en el sedimento marino de la Comuna de Bajo Alto mediante Espectroscopia de Absorción Atómica utilizando generación electroquímica de especies volátiles como técnica de introducción de muestra.

\section{Materiales y métodos}

Como patrón de referencia se utilizó una muestra certificada PACS-2 (National Research Council of Canadá). Para el análisis se procedió a recolectar $1 \mathrm{Kg}$ de muestras de sedimento marino tomadas de seis puntos distintos pertenecientes a la playa de Bajo Alto, cantón El Guabo, provincia de El Oro, cercano a las cuencas de los Ríos Pagua y Jubones que rodean al sitio de estudio. Posteriormente, estas se homogenizaron, luego se pesó $1 \mathrm{~g}$ de muestra en tubos de ensayo previamente lavados con una solución de ácido nítrico - agua (HNO3-H2O) proporción (1:1).

Se agregó $2 \mathrm{ml}$ de ácido clorhídrico $(\mathrm{HCl})$ y $6 \mathrm{ml}$ de ácido nítrico (HNO3) concentrados en proporción 1:3. La adición de los ácidos se debe hacer teniendo en cuenta la precaución de que los tubos de ensayo se encuentren sobre un baño de hielo. Luego se calentaron las muestras en un termoreactor a $150^{\circ} \mathrm{C}$ durante 1 hora.

Una vez transcurrido el tiempo se dejaron enfriar a temperatura ambiente, para proceder a centrifugar las mismas 
durante 5 minutos. Después se filtraron en un balón aforado cada una de las muestras para remover los sólidos suspendidos y llevar a un volumen de 50 ml para la cuantificación de arsénico mediante generación electroquímica acoplada a espectroscopía atómica.En cuanto a la generación electroquímica de hidruros (EcHG), se utilizó una celda de vidrio, con los habituales tres electrodos. Se utilizó platino $(\mathrm{Pt})$, oro $(\mathrm{Au})$, carbón vítreo reticulado $(\mathrm{RGC})$, plomo (Pb) y Oro/mercurio (Au/Hg)) como electrodos de trabajo o cátodos, una bobina de platino como contra-electrodo y un electrodo de referencia de calomelanos saturado (ECS). Esta celda fue conectada al espectrofotómetro de absorción atómica (Figura 1).

Se utilizó ácido sulfúrico (H2SO4) como medio electrolítico. Este fue seleccionado a partir de diversos estudios reportados en la literatura sobre catolitos para la Generación Electroquímica de Hidruros (EcHG): el ácido nítrico (HNO3) actúan como depolarizante catódico y suprime la formación de hidrógeno reduciendo así la formación de especies volátiles de arsénico (M. Sáenz, L. Fernández, J.R. Dominguez, J. Alvarado, Electrochemical Generation of Lead Volatile Species as a Method of Sample Introduction for Lead Determination by Atomic Absorption Spectrometry, Electroanalysis. 22 (2010) 2842 - 2847).

Figura 1. Celda electroquímica acoplada al espectrofotómetro de absorción atómica

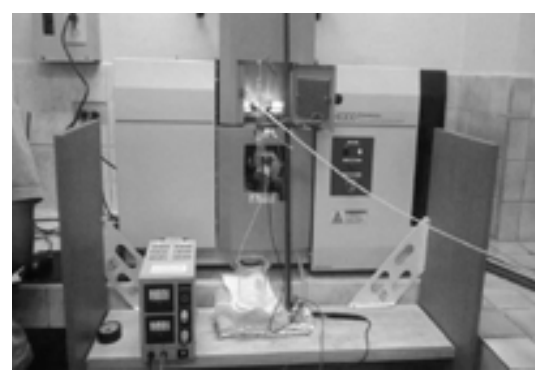

Elaboración propia: Romero y col (2014)

Para la curva de calibrado se utilizaron soluciones que contenían concentraciones conocidas de arsénico (As). Posteriormente se aplicó una corriente electroquímica constante para generar los productos gaseosos de interés que luego fueron introducidos en la celda de atomización de cuarzo en virtud de un flujo de argón (Ar), a través de un separador de gas líquido, para disociación térmica y detección mediante espectroscopía atómica. Un resumen de las condiciones iniciales de trabajo se presenta en la Tabla 1.

\section{Resultados}

La Figura 2, muestra el efecto de los diferentes electrodos
Tabla 1. Condiciones de trabajo para la generación electroquímica de hidruro volátil de arsénico y su determinación en sedimento marino mediante espectroscopía atómica

\begin{tabular}{|ll|}
\hline \multicolumn{1}{|c|}{ Parámetros } & $\begin{array}{l}\text { Carbón vítreo reticulado (RGC), } \mathrm{Pb}, \mathrm{Au}, \\
\mathrm{Pt}, \mathrm{y} \mathrm{Au} / \mathrm{Hg}\end{array}$ \\
\hline Materiales Catódicos & 0.6 \\
\hline Superficie de cátodo $\left(\mathrm{cm}^{2}\right)$ & Platino (Alambre en forma de espiral) \\
\hline Material del Ánodo & $0.6-0.8$ \\
\hline Corriente Electroquímica (A) & $\mathrm{H}_{2} \mathrm{SO}_{4} 0.5 \mathrm{M}$ \\
\hline Electrolito Soporte $(\mathrm{M})$ & 50 \\
\hline Flujo de Argón $\left(\mathrm{mLmin}^{-1}\right)$ & \\
\hline
\end{tabular}

Elaboración propia: Romero y col (2014)

de trabajo utilizados como material cátodico en la señal generada a partir de una solución de 0.1 ppm de Arsénico y $0.5 \mathrm{M}$ de $\mathrm{H} 2 \mathrm{SO} 4$, aplicando una velocidad de flujo $50 \mathrm{~mL} /$ min y una corriente electrolítica 0.8A.

Figura 2. Efecto del material del cátodo en la señal generada desde una solución de $0.1 \mathrm{ppm}$ de As y $0.5 \mathrm{M}$ de $\mathrm{H} 2 \mathrm{SO} 4$, velocidad de flujo $50 \mathrm{~mL} / \mathrm{min}$, corriente electrolítica 0.8A. a) $\mathrm{Pt}$, b) $\mathrm{Au}$, c) $\mathrm{Pb}$ d) $\mathrm{RGC}$, e) $\mathrm{Au} / \mathrm{Hg}$

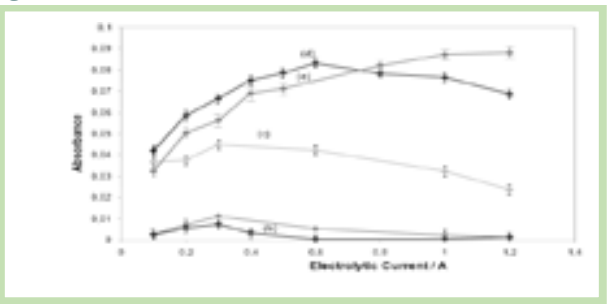

Elaboración propia: Romero y col (2014)

Con el método electroquímico espectroscópico optimizado se cuantificó la concentración de arsénico en las muestras de sedimento marino de Bajo Alto, donde se pudo determinar un valor de $0.05 \mathrm{mg} / \mathrm{Kg}$ este metal.

\section{Discusión}

La Figura 2 permite observar que cuando se utilizó como electrodo de trabajo de Pt y Au (Curva 2a y 2b, respectivamente) la señal de absorbancia primero aumenta entre 0.1 y 0.3A, alcanzando un valor máximo a 0.01, luego disminuye a valores de corriente más bajos. La disminución muy pronunciada de la corriente puede explicarse teniendo en cuenta que los cátodos de Pt y Au presentan mayor tendencia a la generación de hidrógeno molecular $(\mathrm{H} 2)$ en sobre potenciales bajos, así como tienen una mayor capacidad de adsorción de hidrógeno dentro de la superficie, lo que sugiere que los sitios activos en la superficie del cátodo no están operativos para la generación electroquímica de arsina.

En el cátodo de Pb (Figura 2c), la absorbancia aumentó ligeramente entre 0.1A y 0.3A y luego disminuyó entre 0.6 y 1.2A. Sobre el cátodo de Pb, tanto la generación de hidrógeno así como la generación de Plumbano ( $\mathrm{PbH} 4)$ puede

\section{$14 \quad$ Cumbres}


afectar al rendimiento de arsina (M. Sáenz, L. Fernández, J. Domínguez, J. Alvarado. Electrochemical generation of volatile lead species using a cadmium cathode: Comparison with graphite, glassy carbon and platinum cathodes, Spectrochimica Acta Part B 71-72 (2012)107-111), lo que puede explicar la disminución de absorbancia.

En carbón vítreo reticulado (RGC) (Figura 2d), la absorbancia aumenta de 0.1 a 0.6A y luego disminuye lentamente. Comparando el cátodo de carbón vítreo reticulado con el cátodo de plomo, puede verse en el gráfico que la caída de la absorbancia es menos drástica en el primero que en el segundo. En el cátodo de Au/Hg (Figura 2e), la absorbancia aumentó gradualmente con la corriente, logrando mayores valores de absorbancia entre 0.8 y 1.2 que los obtenidos en el carbón vítreo reticulado (RGC), lo que sugiere que el cátodo Au/Hg podría tolerar mejor las interferencias que los otros cátodos.

\section{Conclusiones}

1. Se desarrolló una metodología para la determinación de arsénico por Espectroscopia de Absorción Atómica, utilizando la generación electroquímica de hidruro de arsénico (arsina AsH3) sobre un cátodo de Au/Hg como una técnica de introducción de muestra.

2. El cátodo de Au/Hg es una excelente opción para la generación electroquímica de arsina, debido a su resistencia mecánica, su tolerancia a interferencias y su buena sensibilidad; es resistente y confiable, y por lo tanto puede utilizarse durante largos períodos de tiempo sin deterioro del rendimiento. Permite trabajar sobre potenciales de hidrógeno adecuados para la generación de la especie volátil a partir de As (III).
3. En conclusión, los sedimentos marinos de la comuna de Bajo Alto no se encuentran contaminados por arsénico (As) ya que la concentración de este metal fue de $0.05 \mathrm{mg} / \mathrm{Kg}$, mientras que los límites permitidos de acuerdo a la Organización Mundial de la Salud son de $5 \mathrm{mg} / \mathrm{Kg}$.

\section{Referencias bibliográficas}

- Laborda, F., Górriz, M., Bolea, E., Castillo, R. (2007, 743751). Electrochemical Hydride generation as a simple-introduction technique in atomic spectrometry. Fundamentals interferences and applications.

- Sáenz, M., Fernández, L., Dominguez, J., Alvarado, J. (2010, 2842-2847). Electrochemical Generation of Lead Volatile Species as a Method of Sample Introduction for Lead Determination by Atomic Absorption Spectrometry. Electroanalysis.

- Sáenz, M., Fernández, L., Domínguez, J., Alvarado, J. (2012, 107-111). Electrochemical generation of volatile lead species using a cadmium cathode: Comparison with graphite, glassy carbon and platinum cathodes. Spectrochimica Acta Part B.

- Zhang, W., Yang, X., Dong, Y., Chu, X. (2010). Application of alkaline mode electrochemical hydride generation for the detection of As and Sb using atomic fluorescence spectrometry.

- Zhang, W., Yang, X., Dong, Y., Chu, X. (2010, 571-578). ApplicatSpectrochimica Acta Part B.

- Zhang, W., Gan, W., Lin, X. (2006, 1316-1321). Development of a new electrochemical hydride generator with tungsten wire cathode for the determination of As and Sb by atomic fluorescence spectrometry. Talanta. 